$$
\begin{gathered}
\text { CONF-9606271--1 } \\
\text { ANL/ET/CP - } 91028 \\
\text { CHEMICAL DECOMPOSITION BY NORMALIZATION } \\
\text { OF MILLIMETER-WAVE SPECTRA* C EIVED } \\
\text { SFP } 191996 \\
\text { OS TI }
\end{gathered}
$$

K. Gopalan* and N. Gopalsami

Department of Engineering

Purdue University Calumet

Hammond, IN 46323

Energy Technology Division

Argonne National Laboratory

Argonne, Illinois 60439

\begin{abstract}
The submitted manuscript has been authored by a contractor of the U.S. Government under contract No. W-31-109-ENG-38. Accordingly, the U.S. Government retains a nonexclusive, royalty-free license to publish or reproduce the published form of this contribution, or allow others to do so, for U.S. Government purposes.
\end{abstract}

DISTRIBUTION OF THIS DOCUMENT IS UNLIMTED

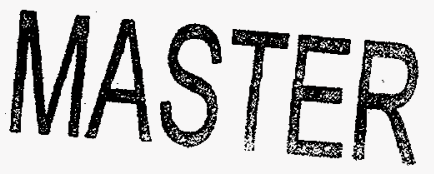

To appear in Proceedings of IEEE Instrumentation and Measurement

Technology Conference, Brussels, Belgium, June 4-6, 1996

*Work supported by U. S. Department of Energy, Office of Nonproliferation and National Security, Office of Research and Development, under Contract W-31-109-ENG-38 


\section{DISCLAIMER}

Portions of this document may be illegible in electronic image products. Images are produced from the best available original document. 


\section{DISCLAIMER}

This report was prepared as an account of work sponsored by an agency of the United States Government. Neither the United States Government nor any agency thereof, nor any of their employees, makes any wartanty, express or implied, or assumes any legal liability or responsibility for the accuracy, completeness, or usefulness of any information, apparatus, product, or process disclosed, or represents that its use would not infringe privately owned rights. Reference herein to any specific commercial product, process, or service by trade name, trademark, manufacturer, or otherwise does not necessarily constitute or imply its endorsement, recommendation, or favoring by the United States Government or any agency thereof. The views and opinions of authors expressed herein do not necessarily state or reflect those of the United States Government or any agency thereof. 
IEEE Instrumentation and Measurement

Technology Conference

Brussels, Belgium, June 4-6, 1996

\title{
Chemical Decomposition by Normalization of Millimeter-Wave Spectra
}

\author{
K. Gopalan ${ }^{1}$ and N. Gopalsami 2 \\ 1 Department of Engineering \\ Purdue University Calumet \\ Hammond, IN 46323 \\ (219) 9892685 \\ E-mail: gopalan@nwi.calumet.purdue.edu \\ 2 Energy Technology Division \\ Argonne National Laboratory \\ Argonne, IL 60439 \\ (708) 2525925 \\ n_gopalsami@qmgate.anl.gov
}

\begin{abstract}
The sharp and distinct absorption spectra of chemicals at low pressures in the millimeter wave range of frequencies become broadened at high pressures. As a result, detecting and quantifying different chemicals at high pressures from the broadened and merged spectra are difficult. This paper proposes a method of decomposition based on the low pressure spectra. Normalized low pressure spectral amplitudes are used as features to train a rieural network. The network is tested using the peak spectra obtained for an unknown plume of chemicals at high pressure. Initial tests conducted on simulated and experimental spectra of selected chemicals show that the decomposition results of the proposed method are dependent on the dominance of the chemicals in the mixture - a characteristic common to conventional methods of decomposition.
\end{abstract}

\section{INTRODUCTION}

Millimeter (mm) wave spectroscopy enables better remote monitoring of airborne chemicals than optical spectroscopy. A $\mathrm{mm}$ wave source signal whose frequency is swept in the range of $220 \mathrm{GHz}-320 \mathrm{GHz}$ is transmitted to the plume of chemicals to be detected. The spectra of the received signal correspond to the absorption of $\mathrm{mm}$ wave energy at frequencies that are characteristic of the chemicals present in the plume. Under ideal conditions and low pressure, each chemical targeted for detection will have sharp spectral (absorption) peaks at frequencies distinct from those of all other chemicals. At high pressure, however, the absorption spectrum of a received signal is complicated because of the following. (a) Due to increase in pressure (above 1 Torr), the spectrum of a molecule becomes broader relative to that at low pressure; as a result, spectra of different molecules may overlap and merge together. (b) Presence of unknown, non targeted chemicals may cause merging and broadening of spectra. (c) Instrumentation noise may contribute to the low-level absorption data and nonuniformly vary the spectral peaks. This paper proposes a method to obtain the composition of certain targeted chemicals from the experimentally observed spectrum of a mixture of chemicals.

\section{ACQUISITION OF EXPERIMENTAL SPECTRA}

The experimental setup used at Argonne for acquiring $\mathrm{mm}$ wave spectroscopic data employs an active $\mathrm{mm}$ wave source with a capability for sweeping the frequency in two ranges of $220-270 \mathrm{GHz}$ and $270-330$ $\mathrm{GHz}$. Using waveguides and an antenna, the $\mathrm{mm}$ wave signal is transmitted into one end of a gas cell containing the chemical mixture to be decomposed. The transmitted signal available at the other end of the gas cell is detected by a hot-electron bolometer. Voltage output of the bolometer is proportional to the power of the detected signal. As the frequency of the $\mathrm{mm}$ wave source is swept in a $2 \mathrm{~s}$ interval, for example, the bolometer output corresponds to the power spectrum of the transmitted (absorbed) signal in the frequency range of the source. A preamplifier filters high frequency noise and provides voltage gain. A 12 bit digitizer is used to acquire the data at the output of the preamplifier. The absorbed signal power in the range of $220-330 \mathrm{GHz}$ is obtained from the combined data acquired for the two frequency ranges. Finally, the percent absorption spectrum is obtained as a ratio:

$\%$ absorption $=100\left(P_{s}-P_{r}\right) / P_{s}$

where 
$P_{s}=$ transmitted signal power with chemical present

$P_{r}=$ transmitted signal power without chemical

\section{FEATURE EXTRACTION AND CHEMICAL DECOMPOSITION}

We note that the effect of high pressure on the $\mathrm{mm}$ wave spectrum of a chemical is to broaden the frequency of absorption while keeping the peak absorption at the same frequency. The broadening effect is given by the Lorentzian shape function as [2]

$S(f)=(K / \pi)\left[f_{L} /\left(f-f_{0}\right)^{2}+f_{L}{ }^{2}\right]$

where

$f_{0}$ is the absorption (center) frequency

$f_{L}$ is the Lorentz line width

$K$ is a constant representing the total strength

Since the peak absorption frequencies for each constituent chemical are maintained, the local maxima in the spectra of a plume of chemicals at high pressure correspond to the combination of the absorption spectra of each chemical at low pressure.

\section{Normalization by Peak Detection}

If $y(n), n=0,1,2, . . N-1$, represents the absorption spectral data for a mixture of chemicals at high pressure, local maxima can be-located by using first and second differences. To reduce the effect of instrumentation noise and the sensitivity of the differentiator, only those peaks that correspond to the frequencies in the low pressure spectra of any of the constituent chemicals are used as elements of a feature vector. Because of the additive behavior of the spectrum of a plume, spectra for a number of plumes are created by adding known combinations of individual, experimentally obtained spectra at high pressure. Peak spectral feature vectors for these synthesized chemical mixtures are used as training inputs to a two-layer backpropagation neural network [3]. The network output for the feature vector for the experimental spectral data of an unknown mixture of the targeted. chemicals corresponds to the composition of the mixture.

For initial testing of this method, absorption spectra in the frequency range of $220-320 \mathrm{GHz}$ for three chemicals, namely, $\mathrm{CH}_{3} \mathrm{Cl}_{35}, \mathrm{CH}_{3} \mathrm{CN}$ and $\mathrm{HC}_{3} \mathrm{~N}$, referred to as $c_{1}, c_{2}$ and $c_{3}$, were used. Spectra of these chemicals were synthesized at atmospheric pressure by applying Lorentzian line shape function for each individual spectrum at low pressure (1 Torr). A combination of the three synthesized spectra at high pressure was added to form a composite (synthetic mixture) at high (atmospheric) pressure as

$p=c_{1} n_{1}+c_{2} n_{2}+c_{3} n_{3}$

where $n_{1}, n_{2}$ and $n_{3}$ are the individual concentrations of $c_{1}, c_{2}$ and $c_{3}$, respectively. We note that the concentration of each chemical is in the ratio of its pressure to the pressure of all the chemicals in the plume. Fig. 1 shows the spectrum of a synthesized composite with $\left[\begin{array}{lll}n_{1} & n_{2} & n_{3}\end{array}\right]=\left[\begin{array}{lll}0.7 & 0.35 & 0.5\end{array}\right]$. The normalized (peak) spectrum of this composite, shown by vertical lines, was obtained by detecting the peaks in the composite and using only those peaks that have corresponding peaks in the spectrum of any of the three chemicals.

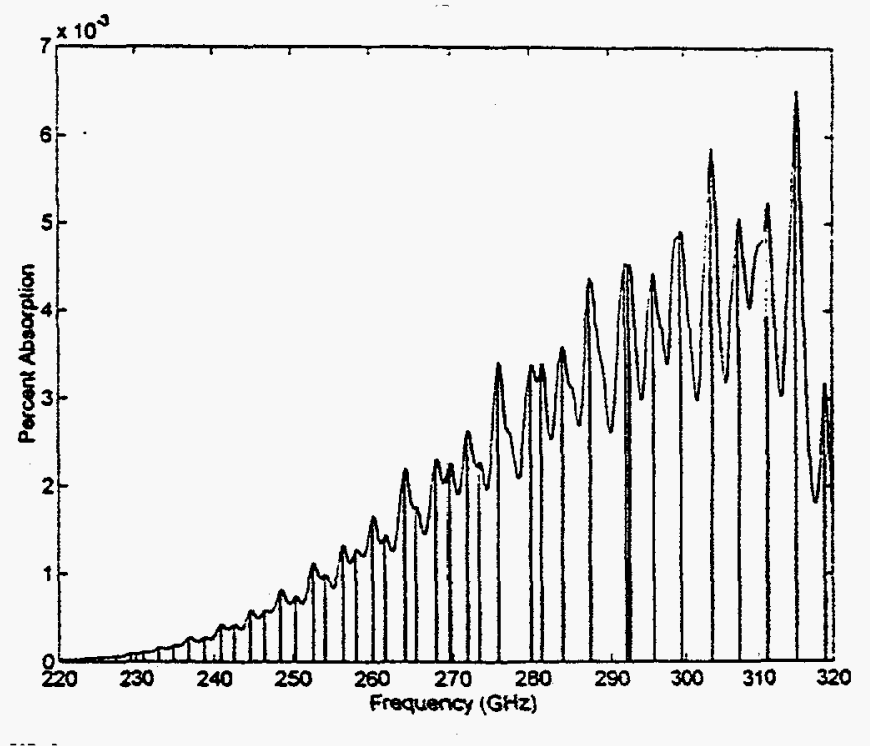

Fig. 1 Spectrum of a synthesized composite and its nomalized peak (low pressure) spectrum

A set of seven known (random) combinations of the three chemicals was used to train a two-layer backpropagation neural network. The normalized peak spectra of the seven composite chemicals were used as input features with their known concentrations as desired outputs. The trained network was tested on two sets of random combinations of the three chemicals. The results are shown in Table I for three methods of feature extraction. The first column, under 'Raw Data', 
shows the results obtained for using the spectral samples of composite chemicals as features with no preprocessing. Features for the Fourier transform method were obtained by treating the spectral data as time-domain samples and taking their discrete Fourier transform. Since the high pressure absorption spectrum, in general, has its peaks broadened, its transform has only low "frequency" components in the Fourier domain. Therefore, only the magnitude of the first 400 points in a 2048-point discrete Fourier transform of each composite spectral signal was used as the feature vector. In all three cases the same twolayer neural network was used with 2500 training steps.

Table I

a. Synthesized Composite Mixture 1

$\begin{array}{llll}\begin{array}{l}\text { Synth. } \\ \text { Mixture }\end{array} & \text { Raw Data } & \begin{array}{l}\text { Derivative } \\ + \text { Peaks }\end{array} & \begin{array}{l}\text { Fourier } \\ \text { Transform }\end{array} \\ c_{1}=0.70 & 0.6203 & 0.6673 & 0.7461 \\ c_{2}=0.35 & 0.3623 & 0.3994 & 0.3088 \\ c_{3}=0.50 & 0.5049 & 0.4988 & 0.5005\end{array}$

b. Synthesized Composite Mixture 2

$\begin{array}{llll}\begin{array}{l}\text { Synth. } \\ \text { Mixture }\end{array} & \text { Raw Data } & \begin{array}{l}\text { Derivative } \\ \text { + Peaks }\end{array} & \begin{array}{l}\text { Fourier } \\ \text { Transform }\end{array} \\ c_{1}=0.2 & 0.5880 & 0.5990 & 0.1964 \\ c_{2}=0.3 & 0.2432 & 0.4135 & 0.2613 \\ c_{3}=0.4 & 0.3859 & 0.5459 & 0.3798\end{array}$

As can be seen from the above table for two different synthesized mixtures, the peak detection method gives better decomposition results compared to using raw data. The Fourier transform-based feature vector, while taking a slightly higher computational effort, also yields results comparable to peak detection method for the first mixture. For the second mixture of $\left[n_{1} n_{2} n_{3}\right]=\left[\begin{array}{ll}0.2 & 0.3\end{array}\right.$ 0.4], however, decomposition results based on the Fourier transform samples are better than the peak detection method. This may be attributed to the dominant low pressure spectra of $c_{2}$ as can be seen in the combined spectra of $c_{1}, c_{2}$ and $c_{3}$, with the respective ratios, shown in Fig. $2 a$ and $b$. Although the mixtures are in different ratios, the two spectra have almost identical shape and height; hence, the peak detection method fails to distinguish them clearly. With a 'frequency' resolution of $f_{s} / 2048$, the discrete Fourier transform is better able to identify the component chemicals.

For the second test, individual spectrum of each of three chemicals, $\mathrm{CH}_{3} \mathrm{Cl}\left(\mathrm{Ch}_{1}\right), \mathrm{CH}_{3} \mathrm{CN}\left(\mathrm{Ch}_{2}\right)$, and $\mathrm{CH}_{2} \mathrm{Cl}_{2}\left(\mathrm{Ch}_{3}\right)$, was obtained at a pressure of below $505 \mathrm{mTorr}$ and normalized to 1 Torr. In the absence of known low pressure data (similar to those used in the previous neural network), peak detection was first used on the three spectra to obtain the normalized low pressure spectrum of each chemical. The measured high pressure spectra of the three (at 1 Torr of pressure) were added in random (known) combinations to obtain nine synthesized plumes of chemicals. A neural network similar to the one used for the previous simulated data was trained using (a) the synthesized raw data, (b) the local peak spectral values at the location of the peaks of the normalized low pressure spectrum of each chemical, and (c) the first 500 samples of the magnitude of 4096-point discrete Fourier transform of the synthesized plumes. The trained network in each case, after 2500 iterations, was tested using (a) a synthesized mixture

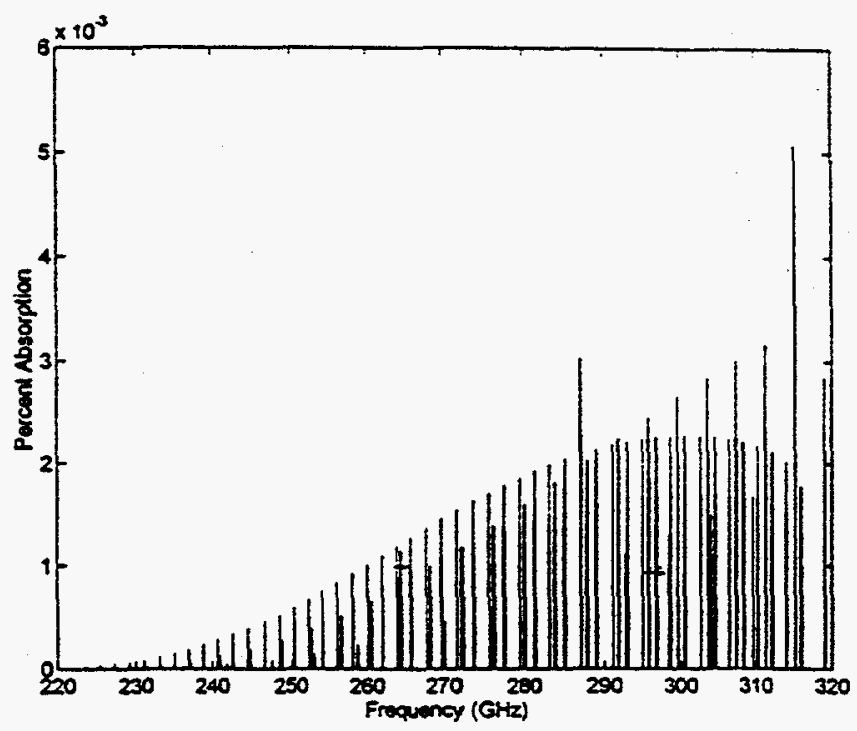

a. $\left.\left[n_{1} n_{2} n_{3}\right]=0.70 .350 .5\right]$

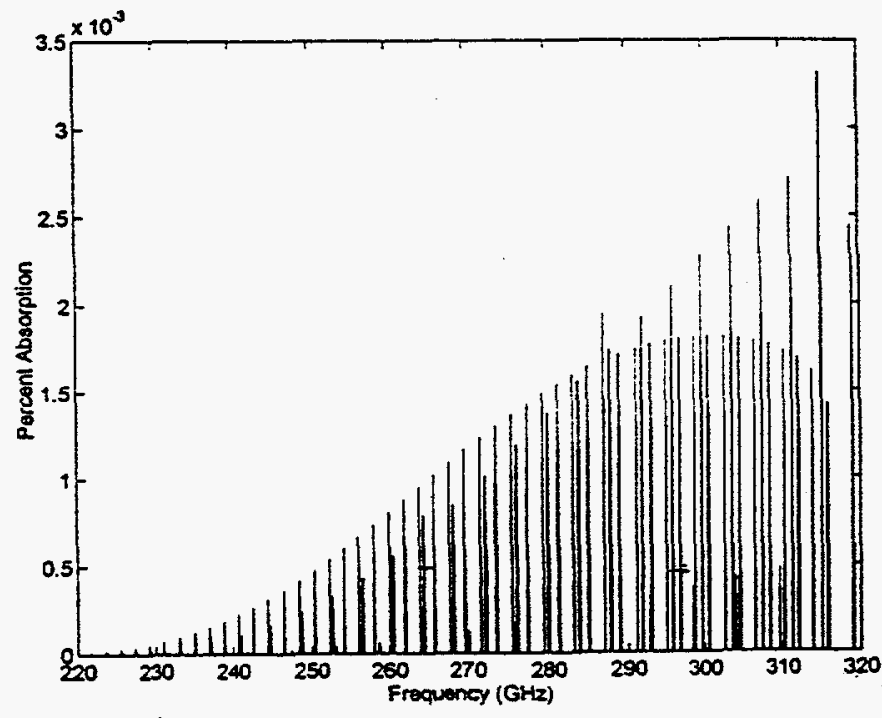

b. $\left[n_{1} n_{2} n_{3}\right]=\left[\begin{array}{lll}0.2 & 0.3 & 0.4\end{array}\right]$

Fig. 2 Peak spectra of $c_{1}, c_{2}$ and $c_{3}$ 
of individual spectra with a concentration of $\left[\mathrm{Ch}_{1} \mathrm{Ch}_{2} \mathrm{Ch}_{3}\right]$ $=\left[\begin{array}{llll}0.2 & 0.3 & 0.4\end{array}\right]$, and (b) an experimental plume with a concentration of $\left[\mathrm{ch}_{1} \mathrm{Ch}_{2} \mathrm{Ch}_{3}\right]=\left[\begin{array}{lll}0.4 & 0.2 & 0.6\end{array}\right]$. We note that the three chemicals, $\mathrm{CH}_{3} \mathrm{Cl}\left(\mathrm{Ch}_{1}\right), \mathrm{CH}_{3} \mathrm{CN}\left(\mathrm{Ch}_{2}\right)$, and $\mathrm{CH}_{2} \mathrm{Cl}_{2}\left(\mathrm{Ch}_{3}\right)$, were at pressures of 400 mTorr, 200 mTorr and $600 \mathrm{mTorr}$, with nitrogen at a pressure of 758.8 Torr to create a total pressure of 760 Torr (atmospheric pressure). The results of the two tests are shown in Table II.

Table II

a. Experimental Composition 1 - Synthesized Mixture of Individual Spectra

$\begin{array}{lllll}\text { Mixture } & \text { Raw Data } & \begin{array}{l}\text { Derivative } \\ \text { + Peaks }\end{array} & \begin{array}{l}\text { Fourier } \\ \text { Transform }\end{array} \\ \mathrm{Ch}_{1}=0.2 & 0.2706 & 0.3809 & 0.1727 & -- \\ \mathrm{ch}_{2}=0.3 & 0.2685 & 0.3767 & 0.2716 \\ \mathrm{ch}_{3}=0.4 & 0.3471 & 0.4759 & 0.3769\end{array}$

b. Experimental Composition 2 - Spectrum of Mixed Chemicals

$\begin{array}{llll}\begin{array}{l}\text { Actual } \\ \text { Mixture }\end{array} & \text { Raw Data } & \begin{array}{l}\text { Derivative } \\ + \text { Peaks }\end{array} & \begin{array}{l}\text { Fourier } \\ \text { Transform }\end{array} \\ \mathrm{Ch}_{1}=0.4 & 0.3101 & 0.4452 & 0.2441 \\ \mathrm{Ch}_{2}=0.2 & 0.3105 & 0.2641 & 0.2241 \\ \mathrm{Ch}_{3}=0.6 & 0.3958 & 0.4511 & 0.8280\end{array}$

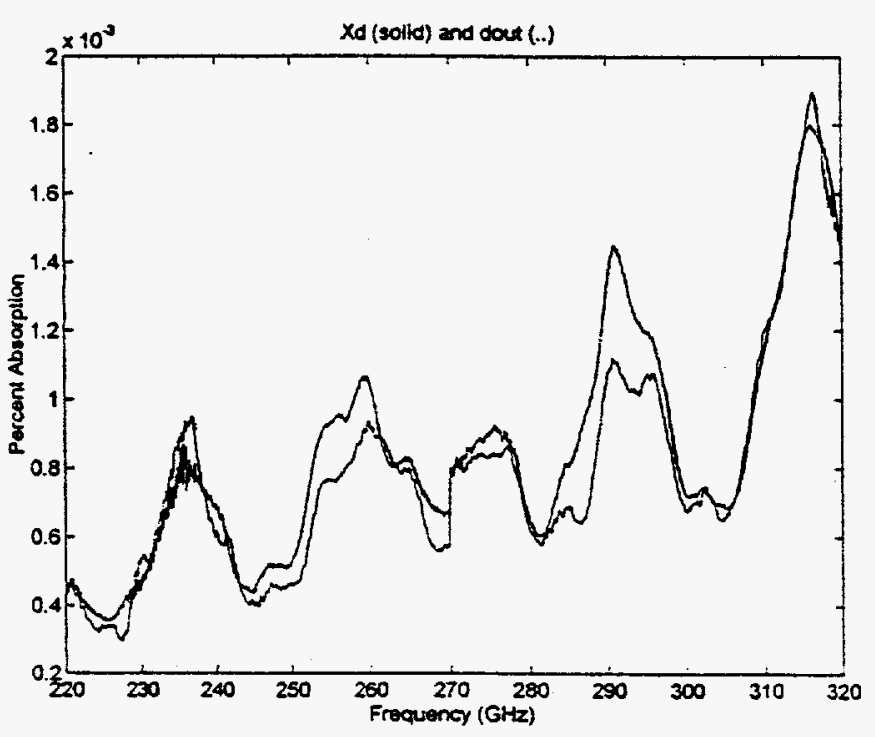

Fig. 3 Experimental spectrum for $\left[\mathrm{ch}_{1} \mathrm{ch}_{2} \mathrm{ch}_{3}\right]=\left[\begin{array}{lll}0.4 & 0.2 & 0.6\end{array}\right]$ and decomposed spectrum, $\left[\mathrm{ch}, \mathrm{Ch}_{2} \mathrm{Ch}_{3}\right]=[0.44520 .26410 .4511]$
As seen from Table lla, the raw data and the Fourier transform samples yield comparable results that are close to the synthesized mixture. Because of the dominant spectrum of $\mathrm{CH}_{3} \mathrm{CN}\left(\mathrm{Ch}_{2}\right)$, however, the peak detection method missed identifying correctly the peak spectral components in the composite mixture thereby resulting in much higher value for $\mathrm{ch}_{1}$. In the case of the experimentally obtained spectrum for the plume (Fig. 3), the decomposition of the chemicals (Table $\| \mathrm{lb}$ ) is better with the peak detection method than with the other two methods. This is because of the proportion of the chemicals used in the experiment: the compositions of $\mathrm{Ch}_{1}$ and $\mathrm{Ch}_{3}$ are high enough to reduce the dominance of $\mathrm{Ch}_{2}$. Raw data and the Fourier transform methods try to match the experimental spectrum of the chemical combination with the spectra of the three used in training. (This type of cluttering of decomposition by a dominant chemical was also observed in the results of chemometric methods.)

\section{CONCLUSION}

A simple feature extraction procedure for the decomposition of individual chemicals from the $\mathrm{mm}$ wave spectral data of a composite mixture has been described. By detecting the peak (local) spectral values in the composite mixture, the spectrum at high pressure is normalized to sharp, low pressure spectrum. With few nonzero values, this sharp spectrum enables easy decomposition of the constituent chemicals. The method is computationally fast and efficient in feature vector size compared with Fourier domain feature extraction and processing. Tests on synthesized and experimental mixtures of composite chemicals show that the method is more suitable for decomposing chemicals if the partial pressures of the chemicals are such that no chemical has a dominant spectral absorption over others.

\section{REFERENCES}

[1] R.L. Poynter and H.M. Pickett, "Submillimeter, Millimeter, and Microwave Spectral Line Catalog." JPL Publication 80-23, Revision 2, 1983.

[2] C. Elachi, Introduction to the Physics and Techniques of Remote Sensing, John Wiley \& Sons, New York, 1987.

[3] MATLAB, High Perfomance Numeric Computation and Visualization Software, The Mathworks Inc., Natick, MA, 1993. 